\section{Structural brain correlates of unconstrained motor activity in people with schizophrenia}

\author{
TOM F. D. FARROW, MICHAEL D. HUNTER, IAIN D. WILKINSON, \\ RUSSELL D. J. GREEN and SEAN A. SPENCE
}

\begin{abstract}
Summary Avolition affects quality of life in chronic schizophrenia.We investigated the relationship between unconstrained motor activity and the volume of key executive brain regions in 16 male patients with schizophrenia.Wristworn actigraphy monitors were used to record motor activity over a $20 \mathrm{~h}$ period. Structural magnetic resonance imaging brain scans were parcellated and individual volumes for anterior cingulate cortex and dorsolateral prefrontal cortex extracted. Patients' total activity was positively correlated with volume of left anterior cingulate cortex. These data suggest that the volume of specific executive structures may affect (quantifiable) motor

behaviours, having further implications for models of the 'will' and avolition.
\end{abstract}

\section{Declaration of interest None.}

Funding detailed in Acknowledgements.

Some modular theories of brain organisation propose a relationship between regional volume and psychological function. In this study we recorded the spontaneous, unconstrained motor behaviour of people with schizophrenia and examined whether such behaviour was related to the volume of key executive brain regions: anterior cingulate (Brodmann area (BA) 32 ) and dorsolateral prefrontal (BA 9 and BA 46) cortices.

The anterior cingulate cortex plays an integrative role in the frontal lobes, translating intentions into actions (Paus, 2001). It contributes to motor control, complex executive processing, vigilance, arousal and 'drive' (Spence \& Frith, 1999). Together with its involvement in autonomic functioning, these processes suggest that the anterior cingulate cortex plays a pivotal role in overall behavioural control
(Devinsky et al, 1995), particularly in goaldirected behaviours (Spence \& Frith, 1999). Conversely, anterior cingulate cortex lesions are associated with reduced spontaneous behaviour and attention, as manifest in akinetic mutism (Devinsky et al, 1995).

The role of the medial prefrontal cortex (including anterior cingulate cortex) in schizophrenia has been much investigated. Structurally there is evidence of abnormal medial prefrontal gyral patterns and cytoarchitecture, whereas functionally there is evidence of reduced perfusion in the psychomotor poverty sub-syndrome (alogia, flatness of affect and decreased spontaneous movement (Liddle et al, 1992). A negative correlation was found between patients' poverty scores and left ventro-medial prefrontal grey matter density (Chua et al, 1997).

Dorsolateral prefrontal cortex, particularly BA 9 and BA 46, has been implicated in behavioural response selection tasks, including finger movement (Hunter et al, 2004), random number generation and verbal fluency, all requiring the individual to generate novelty. Therefore, while anterior cingulate cortex is implicated in the quantity of motor activity performed by the organism, dorsolateral prefrontal cortex regions are implicated in behavioural complexity (e.g. how novel the response patterns are that emerge) (Spence $\&$ Frith, 1999).

We hypothesised that in schizophrenia the extent of motor activity over a prolonged period $(20 \mathrm{~h})$ might be constrained by anatomical features of the frontal executive system, specifically the anterior cingulate cortex (BA 32) and dorsolateral prefrontal cortex (BA 9/46).

\section{METHOD}

Sixteen right-handed patients with DSMIV schizophrenia (American Psychiatric Association, 1994) gave written informed consent and participated in this study, approved by the North Sheffield Research Ethics Committee. All participants were community-based out-patients, but for monitoring purposes were admitted to a psychiatric ward for $24 \mathrm{~h}$. Participants had a mean age of 36 years (s.d. $=8$ ), a mean illness duration of 14 years (s.d. $=8$ ), a mean score on the Scale for the Assessment of Negative Symptoms (SANS; Andreasen, 1985) of 11.5 (s.d.=2.7), a Scale for the Assessment of Positive Symptoms (SAPS; Andreasen, 1985) score of 3.6 (s.d.=2.1) and a Beck Depression Inventory (BDI; Beck et al, 1996) score of 9.5 (s.d.=10.3). Eleven patients were taking oral atypical antipsychotic medication (olanzapine, 6; clozapine, 4; quetiapine, 1), one was taking oral typical medication (sulpiride) and four were receiving typical depot medication (flupenthixol decanoate, 2; fluphenazine decanoate, 1; zuclopenthixol decanoate, 1). Patients underwent a structural magnetic resonance imaging (MRI) scan on a 1.5 Tesla system (Eclipse, Philips Medical Systems, Ohio, USA) using a three-dimensional acquisition technique (RF-spoiled FAST; repetition time $=15 \mathrm{~ms}$; echo time $=4.4 \mathrm{~ms}$; acquisition matrix $=$ $256 \times 256 \times 190$ yielding a voxel size of $1 \mathrm{~mm}^{3}$ ) which produced a $\mathrm{T}_{1}$-weighted volume dataset covering the entire brain. Scans were pre-processed using voxel-based morphometry with statistical parametric mapping (SPM2) (Wellcome Department of Imaging Neuroscience, London). Smoothed grey matter segmented maps were parcellated using masks created with WFP_PickAtlas v1.02 (Maldjian et al, 2003; see data supplement to online version of this paper). Volumes of anterior cingulate cortex and dorsolateral prefrontal cortex grey matter regions were obtained for each participant. Following the scan, participants wore an 'Actiwatch' (Cambridge Neurotechnology, UK) measuring their cumulative activity over a $20 \mathrm{~h}$ period. The Actiwatch is a wrist-worn device containing a miniature uniaxial accelerometer which produces a digital integration of the amount and duration of all movement over $0.05 \mathrm{~g}$. As an indicator of normal daytime activity, a study of 107 healthy 16- to 19 year-old adolescents recorded mean Actiwatch readings of 162565 (s.d.=68620) (a dimensionless measure) over a $24 \mathrm{~h}$ period (Nancy Butte, personal communication, 2004). We ran patient-wise parametric bivariate correlations between total motor activity and volumes of bilateral 
anterior cingulate cortex and dorsolateral prefrontal cortex.

\section{RESULTS}

Over $20 \mathrm{~h}$ the mean Actiwatch reading was 106722 (s.d.=36 553). The mean regional grey matter volume was $4.7 \mathrm{ml}$ (s.d.=0.6) for left anterior cingulate cortex, $5.0 \mathrm{ml}$ (s.d. $=0.4)$ for right anterior cingulate cortex, $5.3 \mathrm{ml}$ (s.d.=1.0) for left dorsolateral prefrontal cortex and $4.4 \mathrm{ml}$ (s.d. $=0.6$ ) for right dorsolateral prefrontal cortex. There were no correlations between patients' ages or durations of illness (which are themselves of course highly positively correlated) or SANS scores and anterior cingulate cortex or dorsolateral prefrontal cortex volumes. There were negative correlations between patients' ages and durations of illness and their total activity measures $(r=-0.549, \quad P=0.034$ and $r=-0.621$, $P=0.014$, respectively). There was a negative correlation between SANS avolition score and Actiwatch-measured motor activity $(r=-0.52, P=0.047)$, although this result should be interpreted in the light of the ordinal nature of the SANS and small variance in our patients' avolition scores (range 2-4). There were no correlations between chlorpromazine-equivalent drug doses or BDI scores and motor activity. Total activity over $20 \mathrm{~h}$ was positively correlated with volume of the left anterior cingulate cortex $(r=0.487, \quad P=0.028$; Fig. 1). There were no significant correlations between cumulative Actiwatch activity and right anterior cingulate cortex or left or right dorsolateral prefrontal cortex volumes $(P>0.3)$.

\section{DISCUSSION}

In these people with schizophrenia, motor activity over a $20 \mathrm{~h}$ period was correlated with the volume of left anterior cingulate cortex, a finding supportive of our central hypothesis that anatomy may constrain function, in this case spontaneous motor behaviour. In particular, it was the anterior cingulate cortex rather than the dorsolateral prefrontal cortex that was implicated, consistent with a methodology that measures 'volume' of motor behaviour, without regard to its complexity. This finding is congruent with the role of anterior cingulate cortex in the emergence of motor acts, and its relative dysfunction in psychomotor poverty (Liddle et al, 1992; Chua et al,

TOM F. D. FARROW, PhD, MICHAEL D. HUNTER, MRCPsych, Sheffield Cognition and Neuroimaging Laboratory (SCANLab), Department of Academic Clinical Psychiatry; IAIN D. WILKINSON, PhD, Department of Academic Radiology; RUSSELL D. J. GREEN, MBChB, SEAN A. SPENCE, MD, MRCPsych, SCANLab, Department of Academic Clinical Psychiatry, University of Sheffield, UK

Correspondence: DrTom F. D. Farrow, SCANLab, Department of Academic Clinical Psychiatry, University of Sheffield, The Longley Centre, Northern General Hospital, Norwood Grange Drive, Sheffield S5 7JT,UK. E-mail: t.f.farrow@sheffield.ac.uk

(First received 14 September 2004, final revision 4 March 2005, accepted 16 March 2005)

1997) and akinetic mutism (Devinsky et al, 1995).

In terms of methodology, ours is the first study of regional brain volume in schizophrenia to use an automated measure of spontaneous motor activity. This novel use of an actigraphy monitor as an objective, scalar measure of bodily movement, as opposed to the more usually quoted, subjective, ordinal measure of avolition (in the SANS), has the potential to augment validity in clinical studies and certainly detects greater between-subject variance. This may be particularly useful in patient assessments where change is anticipated (as with behavioural or pharmacological interventions).

We must be cautious in extrapolating our finding to community, ambulatory patients as our study concerned patients who spent the days in question on a psychiatric ward. Nevertheless, our data do offer the intriguing possibility that anatomy and function are related with respect to spontaneous, unconstrained motor activity. Of course, there is a final caveat in that we do not know the extent to which measured behaviour is purposeful (Spence \& Frith,

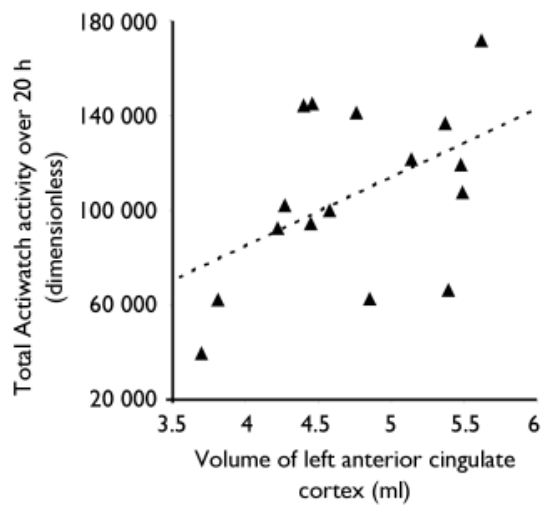

Fig. I Positive correlation in 16 patients with chronic schizophrenia between their total cumulative activity over $20 \mathrm{~h}$ and left anterior cingulate cortex (Brodmann area 32) volumes.
1999), but this is the subject of ongoing work (Hunter et al, 2004).

In summary, our findings suggest that the volume of the left anterior cingulate cortex in people with chronic schizophrenia correlates with an objective measure of their unconstrained motor activity.

\section{ACKNOWLEDGEMENTS}

This study was funded by an investigator-led grant awarded to S.A.S. by Cephalon UK. M.D.H. is supported by the Wellcome Trust. We thank Professor Nancy Butte, Baylor College of Medicine, Texas, USA for normative Actiwatch data.

\section{REFERENCES}

American Psychiatric Association (1994) Diagnostic and Statistical Manual of Mental Disorders (4th edn) (DSM-IV). Washington, DC. APA

Andreasen, N. C. (1985) Positive vs. negative schizophrenia: a critical evaluation. Schizophrenia Bulletin, II, 380-389.

Beck, A.T., Steer, R. A. Q. \& Brown, G. K. (1996) Manual for Beck Depression Inventory-II. San Antonio, TX: Psychological Corporation

Chua, S. E., Wright, I. C., Poline, J.-B., et al (1997) Grey matter correlates of syndromes in schizophrenia. A semi-automated analysis of structural magnetic resonance images. British journal of Psychiatry, 170 , 406-410.

Devinsky, O., Morrell, M. J. \& Vogt, B. A. (1995) Contributions of anterior cingulate cortex to behaviour. Brain, I18, 279-306.

Hunter, M. D., Green, R. D. J., Wilkinson, I. D., et al (2004) Spatial and temporal dissociation in prefrontal cortex during action execution. Neuroimage, 23. $\mid 186-1191$.

Liddle, P. F., Friston, K. J., Frith, C. D., et al (1992) Patterns of cerebral blood flow in schizophrenia. British Journal of Psychiatry, 160, 179-186.

Maldjian, J. A., Laurienti, P. J., Burdette, J. H., et al (2003) An automated method for neuroanatomic and cytoarchitectonic atlas-based interrogation of $\mathrm{fMRI}$ data sets. Neuroimage, 19, 1233-1239.

Paus, T. (200I) Primate anterior cingulate cortex: where motor control, drive and cognition interface. Nature Reviews Neuroscience, 2, 417-424.

Spence, S. A. \& Frith, C. D. (1999) Towards a functional anatomy of volition. Journal of Consciousness Studies, 6, II-29. 